\title{
Optical Order Parameter of Dichroic Azo-Dyes in Liquid Crystalline Side-Chain Polysiloxanes
}

\author{
E. Chrzumnicka, D. Bauman \\ Faculty of Technical Physics, Poznań University of Technology \\ Nieszawska 13A, 60-965 Poznań, Poland \\ AND J. JADŻYN \\ Institute of Molecular Physics, Polish Academy of Sciences \\ Smoluchowskiego 17, 60-179 Poznań, Poland \\ (Received June 6, 2002; revised version September 11, 2002) \\ Polarized light absorption spectra of seven dichroic azo-dyes dissolved in \\ mesogenic side-chain polysiloxanes were recorded as a function of tempera- \\ ture. On the basis of the spectra the optical order parameter of the dyes was \\ determined. The influence of the dye on the phase transition temperatures \\ of polysiloxanes was examined. The results obtained for polysiloxanes were \\ compared with the data for low molecular weight liquid crystal.
}

PACS numbers: 61.41.+e, 61.30.Gd, 64.70.Md

\section{Introduction}

In recent years thermotropic liquid crystalline polymers (LCPs) have been the subject of much research because these substances combine the viscoelastic properties specific for polymers with the well-known properties of low molecular weight liquid crystals in a single material [1-3]. This combination is particularly important for side-chain LCPs [4], where relatively mobile mesogenic side groups may be oriented by means of external forces whereas the polymer backbone provides a suspending matrix. The polymer high viscosity then allows the induced order to be stored by rapid cooling the sample to below the glass transition temperature, $T_{\mathrm{G}}$. Therefore one of the main applications of side-chain LCPs 
will be in optical information data storage. It is also possible to use these substances in electro-optical display devices, waveguides in optical communications, selective filters and reflectors. Moreover, side-chain LCPs can be utilized as the well-orienting solvents to study the direction-dependent molecular properties of the dissolved guest molecules [5] ("guest-host" effect [6]). On the other hand, the guest molecules can be used as probes to reflect the orientational properties and dynamics of polymeric host medium $[7,8]$.

Finally, some dopant molecules oriented in side-chain LCPs give possibility to use such systems in nonlinear optics. It is known that for second order nonlinear processes, such as second harmonic generation or the nonlinear electro-optical effect, it is necessary to operate with the medium with some degree of order [4]. Side-chain LCPs fulfil this requirement. If, additionally, the dopant with high value of the second order hyperpolarizability $\beta$ is chosen, one is able to obtain the system with nonlinear coefficients (at optical frequencies) much larger than those found in inorganic single crystals. The compounds with high $\beta$-value should be searched among the molecules exhibiting large changes of dipole moment at $\pi \rightarrow \pi^{*}$ electronic transition from the ground state to the excited state [4]. Molecules fulfilling this condition are the so-called charge transfer (CT) molecules. Typical examples of such CT-molecules are azo-dyes molecules. They possess delocalized electrons in conjugated $\pi$-electron system and the absorption of the visible light by these dyes results from a $\pi \rightarrow \pi^{*}$ charge transfer transition, the direction of which is mostly parallel to the molecular plane [9]. Therefore, azo-dyes dissolved in side-chain LCPs seem to be ideal nonlinear media. It is also possible to synthesize copolymers containing both mesogenic and azo-dye units in their side chains [4]. This gives a possibility of greater dye concentrations in the liquid crystalline polymeric material to be used and higher optical contrast ratio to be achieved.

In this paper we have presented the results of the study of the orienting behaviour of some dichroic azo-dyes dissolved in polysiloxanes with mesogenic side groups. We would like to check the influence of the molecular structure of the dye on its order parameter as well as the effect of the dichroic dye presence on the mesophase range of the matrix. The results obtained have been compared with the data for the low molecular weight liquid crystal having the molecular structure similar to that of side groups.

\section{Experimental}

Two mesogenic side-chain siloxane polymers with different spacer length (LCP 1 and LCP 2) and low molecular weight liquid crystal (LC 1) were used as host matrices. LCPs were synthesized at the Warsaw Academy of Agriculture (Faculty of General Chemistry), as described in the literature [10], via hydroxylation of an alkene type mesogen [4-(5-alkenyloxy)-benzoic acid 4'-methoxypheny] ester] with poly-(methylhydrogensiloxane) (MERCK). LC 1 was synthesized at the Military University of Technology in Warsaw (Institute of Chemistry). The 
TABLE I

Molecular structure and mesophase ranges of liquid crystal materials investigated.

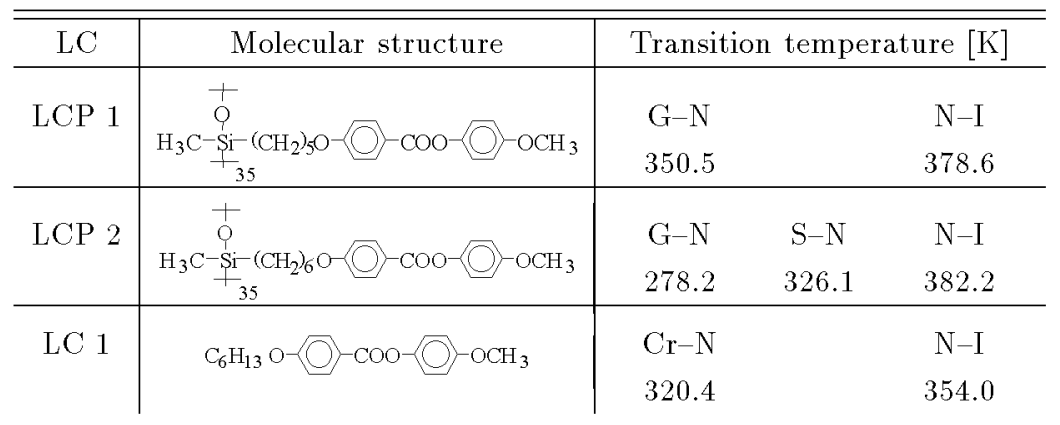

molecular structure of these substances and their mesophase range are given in Table I.

As guest species seven dichroic azo-dyes with various molecular sizes and shapes were employed. The molecular structure and approximate values of the molecular length to breadth ratio, $l / d$ for the dyes investigated are gathered in Table II. $l / d$ values were estimated from the known bond lengths and molecular configurations [11], including van der Waals radii of the terminal atoms [12]. All the dyes were synthesized and chromatographically purified at Łódź University of Technology (Institute of Dyes). They were added to the liquid crystalline materials at a concentration of $0.3 \%$ wt.

The polarized light absorption spectra of the guest-host mixtures were recorded in the visible spectral region by means of a SPECORD M40 spectrophotometer (Carl Zeiss Jena) equipped with neutral polarizers. The measurements were made as a function of temperature using "sandwich" cells of $10 \mu \mathrm{m}$ thickness. The temperature of the cells was regulated and controlled with a practical accuracy of $\pm 0.1 \mathrm{~K}$. After changing the measurement temperature, the cell was left for approximately $30 \mathrm{~min}$ to reach a thermal equilibrium. The macroscopic orientation of the samples was obtained by a treatment of glass surfaces of the cell with polyimide and subsequent rubbing. The molecular alignment in such a cell was parallel to the surfaces, and the quality of orientation was controlled with the aid of a polarizing microscope. The absorption spectra of the dyes dissolved in liquid crystalline matrices were recorded for two positions of the cell, that is, with the orientation axis of the sample at angles of $0^{\circ}$ and $90^{\circ}$ with respect to the polarization plane of the light beam. No reference cell was used. Additionally, the base line spectra for pure host were recorded for the same two positions of the cell. After correction for the base line, two components of absorbance, $A_{\|}$and $A_{\perp}$, as a function of the wavelength were obtained.

The temperatures of the phase transitions, both for pure polymeric and low molecular weight liquid crystals as well as for dye-liquid crystal mixtures, 
TABLE II

Molecular structure and the length to breadth ratio, $l / d$ of dyes investigated.

\begin{tabular}{|c|c|c|}
\hline Dye & Molecular structure & $l / d$ \\
\hline 1 & $\left\langle\bigcirc-\mathrm{N}=\mathrm{N}-\langle\bigcirc\rangle-\mathrm{N}=\mathrm{N}-\langle\mathrm{O}\rangle-\mathrm{N}{ }^{\mathrm{CH}_{3}}{ }_{\mathrm{CH}_{3}}^{\mathrm{CH}_{3}}\right.$ & 2.5 \\
\hline 2 & $\mathrm{~N}-\mathrm{CH}_{3}$ & 2.2 \\
\hline 3 & ) $-\mathrm{N}-\mathrm{C}_{2} \mathrm{H}_{5}$ & 2.0 \\
\hline 4 & $=\mathrm{N}-\langle\bigcirc\rangle-\mathrm{N}^{2}\left\langle_{\mathrm{C}_{2} \mathrm{H}_{4}}^{\mathrm{C}_{2} \mathrm{H}_{5}}-\widehat{O}\right.$ & $2.5^{*}$ \\
\hline 5 & & 2.0 \\
\hline 6 & $\left\langle\mathrm{C}_{2} \mathrm{H}_{4}-\langle\mathrm{O}\right.$ & $2.5^{*}$ \\
\hline 7 & $\mathrm{NO}_{2}-\langle\bigcirc\rangle-\mathrm{N}=\mathrm{N}-\left\langle\bigcirc-\mathrm{N}<_{\mathrm{C}_{2} \mathrm{H}_{5}}^{\mathrm{C}_{2} \mathrm{H}_{5}}\right.$ & 1.7 \\
\hline
\end{tabular}

*assuming the linear conformation of the molecule

were determined with an accuracy of $\pm 0.1 \mathrm{~K}$ by means of a polarizing microscope JENAPOL (Carl Zeiss Jena, Germany) equipped with a heating stage THMSE 600 (Linkam, UK). The observations were made by heating and cooling the probe with the rate of $1 \mathrm{~K} / \mathrm{min}$, and in the vicinity of the phase transitions with the rate of $0.2 \mathrm{~K} / \mathrm{min}$.

\section{Determination of the optical order parameter}

A characteristic feature of a mesophase is the existence of the long-range transitional and/or orientational order, which means that the liquid crystal molecules tend to arrange parallel to each other and parallel to a certain preferred direction, described by the director, $\boldsymbol{n}$. Owing to thermal motion, this direction is an average value in a space or a time. When dichroic dye molecules are dissolved in a liquid crystalline matrix they can adopt the orientation of a host and be reoriented by means of the external field (electric or magnetic) [6]. The measure of the efficiency of the dye molecules orientation along the director is the optical order parameter, $S_{\text {op }}$, defined in terms of the experimentally measured absorbances for light polarized parallel and perpendicularly to $n, A_{\|}$and $A_{\perp}$, by [13]:

$$
S_{\mathrm{op}}=\frac{A_{\|}-A_{\perp}}{A_{\|}+2 A_{\perp}} .
$$


At a molecular level $S_{\mathrm{op}}$ is the order parameter connected with the absorption transition moment [13]:

$$
S_{\mathrm{op}}=\frac{1}{2}\left\langle 3(\boldsymbol{n} \cdot \boldsymbol{\mu} / \mu)^{2}-1\right\rangle
$$

where $\boldsymbol{\mu}$ is the transition dipole moment.

By use of angular momentum theory it can be shown that

$$
S_{\mathrm{op}}=\frac{1}{2}\left(3 \cos ^{2} \delta-1\right) S,
$$

where $\delta$ is the angle between the absorption transition moment and the long axis of the dye molecule, and $S$ is the guest order parameter, defined as [14]:

$$
S=\frac{1}{2}\left\langle 3 \cos ^{2} \theta-1\right\rangle
$$

$\theta$ is here the angle between the molecular long axis and $n$.

In Eq. (4) it is assumed that the distribution function of the dye molecules, $f$, is a function of $\theta$ only, i.e. $f(\theta)$, which is strictly true solely for cylindrically symmetric molecules.

\section{Results and discussion}

Figure 1 shows, as an example, the polarized components of the absorption spectrum, $A_{\|}$and $A_{\perp}$ for dye 5 in LCP 2 in the smectic $(T=301.6 \mathrm{~K}$ ) and

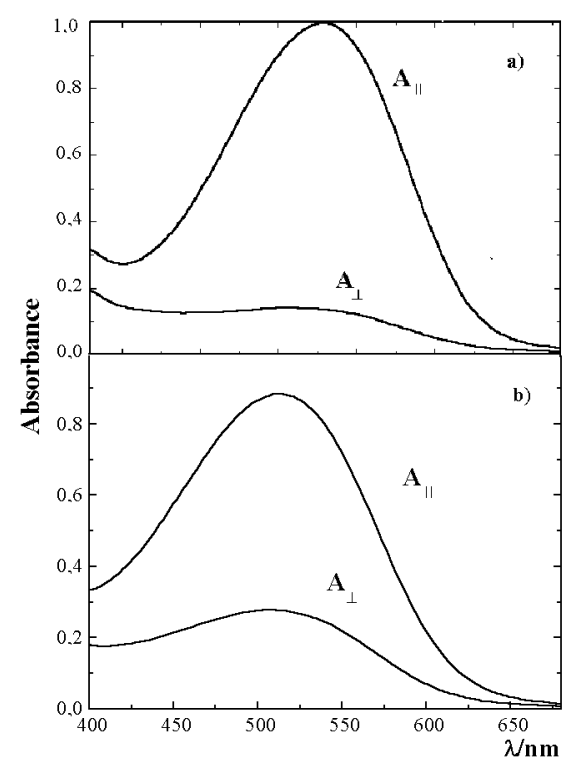

Fig. 1. Polarized light absorption spectra of dye 5 in LCP 2: (a) at $T=301.6 \mathrm{~K}$ (smectic phase) and (b) at $T=355.3 \mathrm{~K}$ (nematic phase). 
TABLE III

Positions of the absorption maximum, $\lambda_{\max }$ of dyes in liquid crystalline materials at $T^{*}=0.93$.

\begin{tabular}{c|c|c|c}
\hline \hline \multirow{2}{*}{ Dye } & \multicolumn{3}{|c}{$\lambda_{\max }[\mathrm{nm}]$} \\
\cline { 2 - 4 } & LCP 1 & LCP 2 & LC 1 \\
\hline 1 & 483 & 483 & 485 \\
2 & $-{ }^{*}$ & 492 & 484 \\
3 & 520 & 515 & 516 \\
4 & 510 & 506 & 512 \\
5 & 495 & 496 & 496 \\
6 & 485 & 486 & 485 \\
7 & 537 & 539 & 542 \\
\hline
\end{tabular}

*dye 2 does not orient in LCP 1

nematic $(T=355.3 \mathrm{~K})$ phases. Such absorption curves are typical of all monoazo dyes investigated - only one absorption band in the visible spectral region related to the intramolecular CT transition is observed. Bis-azo dye 1 has electronic donor character, and in the visible spectral region reveals also only one absorption band connected with the $\pi \rightarrow \pi^{*}$ transition. The position of the band maximum, $\lambda_{\max }$ is different for various dyes and depends on the terminal and lateral substituents as well as slightly on the liquid crystalline host (Table III). The cell thickness and the concentration, chosen in experiment, allow that $A_{\|}<2.0$ and $A_{\perp}>0.1$, therefore the experimental error is minimized. In combination with good samples quality this guarantees a reproducibility of the order parameter $S_{\mathrm{op}}$ with maximal deviations of \pm 0.02 for different samples.

\subsection{Order parameter of dye in liquid crystal matrix}

The value of the order parameter of dichroic dyes in low molecular weight guest-host mixtures depends strongly on the molecular structure of the dye [15-20] and the liquid crystalline host $[16,17,19,21-23]$. The influence of the dye molecular structure and the influence of the type of the mesophase on the dye optical order parameter in liquid crystalline side chain polysiloxanes will be discussed in this section. A comparison will be made of the order parameters of the dyes oriented in the liquid crystalline polymers and in the low molecular weight liquid crystal.

Figures 2, 3 and 4 present the optical order parameter, $S_{\mathrm{op}}$, estimated from the polarized light absorption spectra and Eq. (1), for two chosen azo-dyes in LCP 1, LCP 2 and LC 1, respectively, as a function of the reduced temperature, $T^{*}=T / T_{\mathrm{NI}} . T_{\mathrm{NI}}$ is the average clearing temperature of the guest-host mixture and $T$ is the temperature of the measurement in K. At least three and usually five samples were used to obtain each order parameter, and results presented here are 


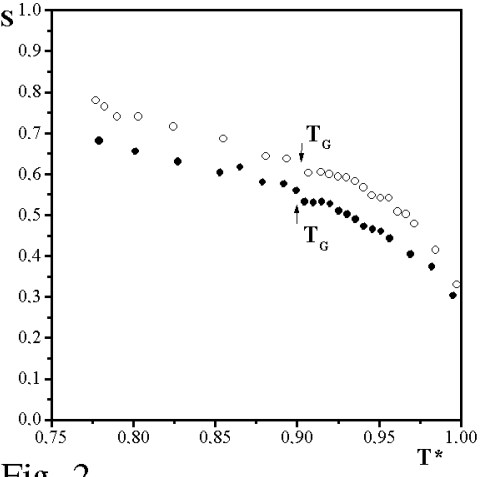

Fig. 2

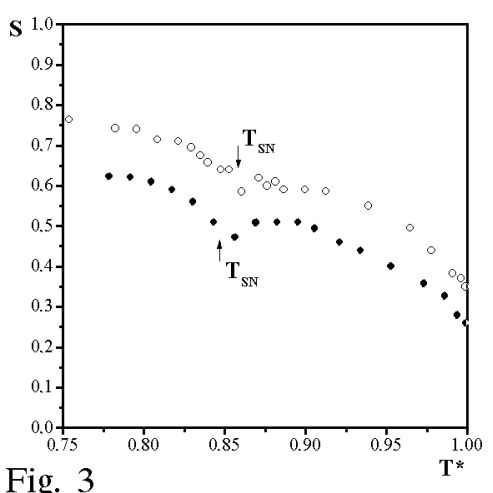

Fig. 3

Fig. 2. Temperature dependence of the optical order parameter for dyes $1(0), 4(\bullet)$ in LCP 1. $T_{G}$ denotes the glassy-nematic phase transition temperature.

Fig. 3. Temperature dependence of the optical order parameter for dyes $1(0), 4(\bullet)$ in LCP 2. $T_{\mathrm{SN}}$ denotes the smectic-nematic phase transition temperature.

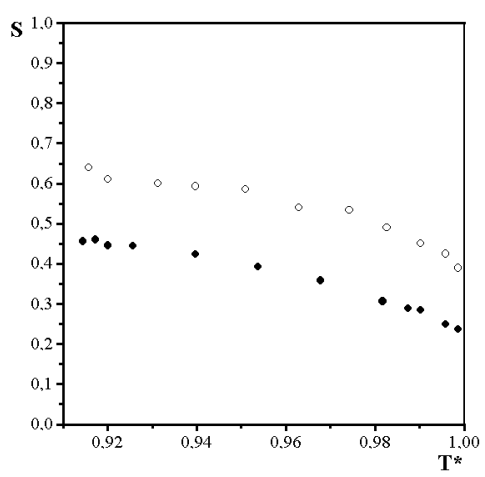

Fig. 4. Temperature dependence of the optical order parameter for dyes $1(0), 4$ in $\mathrm{LC} 1$.

average values. It is seen that the character of changes of the guest order parameter in all the liquid crystal materials in the nematic phase is similar. However, LCP 2 , in the measured temperature range, shows additionally smectic phase, in which $S_{\mathrm{op}}$ increases with the decreasing temperature and reaches high value at the room temperature $\left(T^{*} \approx 0.78\right)$. At the smectic-nematic phase transition the discontinuity in the order parameter is seen very distinctly. In the case of LCP 1 at $T^{*} \approx 0.90$ the transition to the glassy state occurs when the sample is cooling. When the cooling speed is sufficiently slow, in this state the orientation achieved in the nematic phase is continually stored. However, from the results presented in Fig. 2 follows that at the further temperature decreasing the order parameter $S_{\mathrm{op}}$ still rises. This means that the glassy state cannot be strictly treated as a "frozen" state. The change in the orientation of a dye in LCP 1 with the temperature in 
this state can be confirmed by means of polarizing microscopy observations and was also found for other liquid crystalline polymers [7, 24].

In order to compare the optical order parameter for all the dyes investigated in the liquid crystalline materials, the values of $S_{\text {op }}$ at different reduced temperatures corresponding to the various phases are gathered in Tables IV-VI.

\section{TABLE IV}

Optical order parameter, $S_{\mathrm{op}}$ of dyes in LCP 1 at $T^{*}=0.78$ (glassy state), 0.93 (nematic phase) and 0.995 (in the vicinity of clearing point).

\begin{tabular}{c|c|c|c}
\hline \hline \multirow{2}{*}{ Dye } & \multicolumn{3}{|c}{$S_{\mathrm{op}}$} \\
\cline { 2 - 4 } & $T^{*}=0.78$ & $T^{*}=0.93$ & $T^{*}=0.995$ \\
\hline 1 & 0.76 & 0.59 & 0.35 \\
3 & 0.55 & 0.39 & 0.24 \\
4 & 0.66 & 0.49 & 0.30 \\
5 & 0.71 & 0.50 & 0.26 \\
6 & 0.70 & 0.51 & 0.28 \\
7 & 0.53 & 0.37 & 0.22
\end{tabular}

TABLE V

Optical order parameter, $S_{\mathrm{op}}$ of dyes in LCP 2 at $T^{*}=0.78$ (smectic phase), 0.89 and 0.93 (nematic phase) and 0.995 (in the vicinity of clearing point).

\begin{tabular}{c|c|c|c|c}
\hline \hline \multirow{2}{*}{ Dye } & \multicolumn{4}{|c}{$S_{\text {op }}$} \\
\cline { 2 - 5 } & $T^{*}=0.78$ & $T^{*}=0.89$ & $T^{*}=0.93$ & $T^{*}=0.995$ \\
\hline 1 & 0.75 & 0.60 & 0.57 & 0.37 \\
2 & 0.68 & 0.53 & 0.49 & 0.30 \\
3 & 0.75 & 0.59 & 0.56 & 0.36 \\
4 & 0.62 & 0.51 & 0.44 & 0.27 \\
5 & 0.71 & 0.50 & 0.46 & 0.26 \\
6 & 0.73 & 0.56 & 0.51 & 0.27 \\
7 & 0.59 & 0.48 & 0.45 & 0.26
\end{tabular}

From the data presented in Figs. 2-4 and Tables IV-VI results that the value of the optical order parameter of azo-dyes strongly depends on the host matrix, but no regular preference for orientation in low molecular weight liquid crystal or in polysiloxanes is observed. This indicates for the strong intermolecular interactions specific for each guest-host mixtures. The temperature dependence of $S_{\mathrm{op}}$ is different for various dye-liquid crystal mixtures which suggests that the systems investigated are differently resistant to the thermal fluctuations (vibrations, 


\section{TABLE VI}

Optical order parameter, $S_{\mathrm{op}}$ of dyes in $\mathrm{LC} 1$ at $T^{*}=0.93$ (nematic phase) and 0.995 (in the vicinity of clearing point).

\begin{tabular}{c|c|c}
\hline \multirow{2}{*}{ Dye } & \multicolumn{2}{|c}{$S_{\text {op }}$} \\
\cline { 2 - 3 } & $T^{*}=0.93$ & $T^{*}=0.995$ \\
\hline 1 & 0.60 & 0.43 \\
2 & 0.60 & 0.38 \\
3 & 0.49 & 0.30 \\
4 & 0.44 & 0.24 \\
5 & 0.51 & 0.30 \\
6 & 0.50 & 0.29 \\
7 & 0.47 & 0.26
\end{tabular}

librations, and possible internal reorientations of the molecules). The latter observation was made also for these dyes dissolved in another low molecular weight liquid crystal [20].

The data obtained do not allow to draw a general conclusion about the influence of the molecular structure of a dye on its orienting ability. However, some notices can be made. The greatest order parameter of dye 1 in all hosts and at all temperatures indicates that the dye with two azo-linkages can be very well oriented both in low molecular weight liquid crystals and in liquid crystalline polymers. The molecules of dyes 4 and 6 have the same length to breadth ratio as those of dye 1 (see Table II), but only if the linear conformation of the molecules can be assumed. It is however possible that some molecules take the bend conformation due to the presence of $-\mathrm{C}_{2} \mathrm{H}_{4}$ group between $\mathrm{N}$ atom and terminal benzene ring. This decreases the $l / d$ value and influences $S_{\mathrm{op}}$ in all liquid crystalline matrices. The bend conformations cause that steric effects start to play a crucial role. They are the source of the short-range repulsive forces which reduce the guest-host van der Waals interactions by preventing close alignment of the dye and liquid crystal molecules. As a result, the order parameter grows smaller. Similarly, the lateral nitro-group in 3-position (dye 7) can be a steric hindrance for the efficient dye orientation in the liquid crystalline matrix. However, the presence of $\mathrm{Cl}$ atom in the same position seems to improve the efficiency of the dye perfect alignment (compare $S_{\mathrm{Op}}$ for dyes 3 and 5 and dyes 4 and 6 ). The same effect was previously found for other guest-host mixtures [18, 20, 23].

\subsection{Phase transition temperatures of guest-host mixtures}

It was previously found that the addition of a dichroic dye to a low molecular weight liquid crystal changes the phase transition temperatures of the host 
$[13,18-20,23,25,26]$. This effect is especially seen at the nematic-isotropic phase transition, where either a decrease or an increase in the clearing point temperature in the presence of elongated dye molecules was observed. Moreover, in the vicinity of this transition the formation of a two-phase region, in which both nematic and isotropic phases coexist in equilibrium, was ascertained [18-20, 23, 26]. The existence of such a region is in accordance with the thermodynamic laws and the first-order nature of the phase transition for binary mixtures [27]. In the case of polymer the temperatures at which phase transitions take place are usually not so precisely defined as in materials consisting of identical molecules [28, 29] and a phase transitions are spread over some range of temperatures.

Tables VII-IX gather the results of thermal investigations of azo-dyes dissolved in the liquid crystalline polymers and the low molecular weight liquid crystal in the vicinity of the nematic-isotropic phase transition. $T_{\mathrm{N}}$ and $T_{\mathrm{I}}$ denote here the

TABLE VII

Temperature data for nematic-isotropic phase transition of pure LCP 1 and LCP 1 with azo-dyes. All temperatures are given in $\mathrm{K}$.

\begin{tabular}{c|c|c|c|c|c|c}
\hline \hline Dye & $T_{\mathrm{N}}$ & $T_{\mathrm{I}}$ & $T_{\mathrm{I}}-T_{\mathrm{N}}$ & $\Delta T_{\mathrm{N}}$ & $\Delta T_{\mathrm{I}}$ & $\bar{T}_{\mathrm{NI}}$ \\
\hline- & 376.0 & 380.7 & 4.7 & - & - & 378.35 \\
1 & 385.0 & 389.0 & 4.0 & +9.0 & +8.3 & 387.00 \\
3 & 378.2 & 383.7 & 5.5 & +2.2 & +3.0 & 380.95 \\
4 & 387.4 & 393.2 & 5.8 & +11.4 & +12.5 & 390.30 \\
5 & 388.7 & 393.5 & 4.8 & +12.7 & +12.8 & 391.10 \\
6 & 389.5 & 394.7 & 5.2 & +13.5 & +14.0 & 392.10 \\
7 & 389.6 & 394.5 & 4.9 & +13.6 & +13.8 & 392.05
\end{tabular}

TABLE VIII

Temperature data for nematic-isotropic phase transition of pure LCP 2 and LCP 2 with azo-dyes. All temperatures are given in $\mathrm{K}$.

\begin{tabular}{c|c|c|c|c|c|c}
\hline \hline Dye & $T_{\mathrm{N}}$ & $T_{\mathrm{I}}$ & $T_{\mathrm{I}}-T_{\mathrm{N}}$ & $\Delta T_{\mathrm{N}}$ & $\Delta T_{\mathrm{I}}$ & $\bar{T}_{\mathrm{NI}}$ \\
\hline- & 381.1 & 383.2 & 2.1 & - & - & 382.15 \\
1 & 380.7 & 382.9 & 2.2 & -0.4 & -0.3 & 381.80 \\
2 & 380.4 & 382.7 & 2.3 & -0.7 & -0.5 & 381.55 \\
3 & 379.9 & 382.7 & 2.8 & -1.2 & -0.5 & 381.30 \\
4 & 379.4 & 382.0 & 2.6 & -1.7 & -1.2 & 380.70 \\
5 & 380.0 & 383.0 & 3.0 & -1.1 & -0.2 & 381.50 \\
6 & 379.6 & 381.7 & 2.1 & -1.5 & -1.5 & 380.65 \\
7 & 379.7 & 382.8 & 3.1 & -1.4 & -0.4 & 381.25
\end{tabular}


TABLE IX

Temperature data for nematic-isotropic phase transition of LC 1 and LC 1 with azo-dyes. All temperatures are given in $\mathrm{K}$.

\begin{tabular}{c|c|c|c|c|c|c}
\hline \hline Dye & $T_{\mathrm{N}}$ & $T_{\mathrm{I}}$ & $T_{\mathrm{I}}-T_{\mathrm{N}}$ & $\Delta T_{\mathrm{N}}$ & $\Delta T_{\mathrm{I}}$ & $\bar{T}_{\mathrm{NI}}$ \\
\hline- & 354.0 & 354.0 & - & - & - & 354.00 \\
1 & 354.1 & 354.1 & 0.0 & +0.1 & +0.1 & 354.10 \\
2 & 354.0 & 354.0 & 0.0 & 0.0 & 0.0 & 354.00 \\
3 & 353.5 & 353.7 & 0.2 & -0.5 & -0.3 & 353.60 \\
4 & 353.0 & 353.1 & 0.1 & -1.0 & -0.9 & 353.05 \\
5 & 353.4 & 353.6 & 0.2 & -0.6 & -0.4 & 353.50 \\
6 & 353.5 & 353.6 & 0.1 & -0.5 & -0.4 & 353.55 \\
7 & 353.9 & 354.0 & 0.1 & -0.1 & 0.0 & 353.95
\end{tabular}

temperatures at which, at sample heating, the two-phase region begins (the first isotropic drop appears) and ends (the nematic phase entirely disappears), respectively. $\left(T_{\mathrm{I}}-T_{\mathrm{N}}\right)$ is the range of the two-phase region and $\Delta T_{\mathrm{N}}$ and $\Delta T_{\mathrm{I}}$ are the shifts of $T_{\mathrm{N}}$ and $T_{\mathrm{I}}$ for the dye-liquid crystal mixture with respect to these values for pure liquid crystal, whereas $\bar{T}_{\text {NI }}$ denotes the average nematic-isotropic transition temperature. It should be noticed that by heating and cooling the samples some hysteresis was observed: the differences in the phase transition temperatures were $\approx 5 \mathrm{~K}$ for LCP $1, \approx 2 \mathrm{~K}$ for LCP 2 and $\approx 0.2 \mathrm{~K}$ for LC 1 .

For LCP 2 and its mixtures with azo-dyes the smectic-nematic phase transition temperatures were determined additionally. The results are given in Table X. Here $T_{\mathrm{S}}$ and $T_{\mathrm{N}}$ denote the temperatures of, respectively, the beginning and the

TABLE X

Temperature data for smectic-nematic phase transition of LCP 2 and LCP 2 with azo-dyes. All temperatures are given in $\mathrm{K}$.

\begin{tabular}{c|c|c|c|c|c|c}
\hline \hline Dye & $T_{\mathrm{S}}$ & $T_{\mathrm{N}}$ & $T_{\mathrm{N}}-T_{\mathrm{S}}$ & $\Delta T_{\mathrm{S}}$ & $\Delta T_{\mathrm{N}}$ & $\bar{T}_{\mathrm{SN}}$ \\
\hline- & 324.5 & 327.6 & 3.1 & - & - & 326.05 \\
1 & 325.0 & 328.1 & 3.1 & +0.5 & +0.5 & 326.55 \\
2 & 324.6 & 327.7 & 3.1 & +0.1 & +0.1 & 326.15 \\
3 & 322.4 & 325.7 & 3.3 & -2.1 & -1.9 & 324.05 \\
4 & 323.0 & 326.5 & 3.5 & -1.5 & -1.1 & 324.75 \\
5 & 323.6 & 326.8 & 3.2 & -0.9 & -0.8 & 325.20 \\
6 & 323.4 & 326.2 & 3.1 & -1.4 & -1.4 & 324.65 \\
7 & 322.7 & 325.6 & 2.9 & -1.8 & -2.0 & 324.15
\end{tabular}


ending of the phase transition region, and other notations are similar to those given in Tables VII-IX.

From results presented in Table VII follows that azo-dyes added to LCP 1 dramatically influence the temperature of the nematic-isotropic phase transition. The presence of all the dyes causes the large increase in $T_{\mathrm{NI}}$. This is rather unexpected result, considering the small amount of the dye added $(0.3 \% \mathrm{wt})$. However, as the experiment was repeated for various independently prepared samples and within experimental uncertainties the same results were obtained, we should believe that the effect observed must be due to the strong interactions among the polymer and dye molecules. Up to now for low molecular weight liquid crystals some correlation between the shift of $T_{\mathrm{NI}}$ in the presence of a dye and the relation of the guest order parameter with respect to the host order parameter was found: more positive or less negative $\Delta T_{\mathrm{NI}}$ corresponded to an increase in the dye ordering $[13,18-20]$. Taking into account these results, it can be supposed that the side groups of LCP 1 are themselves not able to be well oriented. It can be connected with the perturbation of ordering by the polymer backbone on the one hand and the presence of polymeric molecules with very different numbers of monomers (let us notice that the two-phase region is relatively broad) on the other. The elongated dye molecules added to LCP 1 "stiffen" the side groups and are helpful in the alignment in the preferred direction. As a result of the increase in the degree of order the positive shift of $T_{\mathrm{NI}}$ appears. However, for the mixtures investigated the shift of $T_{\mathrm{NI}}$ is not correlated with the order parameter of the dyes (Table IV). Therefore, it seems that the solution of this problem would be possible when the order parameter of pure LCP 1 and LCP 1 with a dye would be determined by using the same method. This is, however, not very easy to made.

The results of thermal investigations for LCP 2 (Table VIII) are quite different than those for LCP 1: here the influence of the dye on $T_{\mathrm{NI}}$ is not so large. All the dyes added to LCP 2 decrease the nematic-isotropic phase transition temperature and simultaneously cause the broadening of the two-phase region. Similarly as the value of the optical order parameter (Table $\mathrm{V}$ ), the shift of $T_{\mathrm{NI}}$ can be related to the molecular structure of the dye. The least decrease in the clearing temperature is observed for dye 1, whereas the dyes with a heavy terminal group (dyes 4 and 6 ) or a lateral $-\mathrm{NO}_{2}$ group (dye 7 ) distinctly affect $T_{\mathrm{NI}}$ value of the pure LCP 2. Looking at the results for the smectic-nematic phase transition (Table $\mathrm{X}$ ), it is seen that the influence of the dye on the width of the two-phase region is small and in general $T_{\mathrm{SN}}$ decreases in the presence of a dye (except of dyes 1 and 2 ).

The data for the mixtures of the azo-dye with LC 1 reveal that at such low dye concentration in the low molecular weight liquid crystal only very narrow two-phase region in the vicinity of the clearing point can be expected. However, some dyes are able to decrease the nematic-isotropic phase transition temperature about $1 \mathrm{~K}$. One can see that there exists some correlation between the change of $T_{\mathrm{NI}}$ after the dye addition and the order parameter (Table VI). Small order parameter 
of dye 4 is associated with the significant depression of $T_{\mathrm{NI}}$. This dye added to the liquid crystalline matrix causes the disordering of the host molecules and reduces the region of the mesophase. However, dyes 1 and 2 which do not decrease the clearing temperature of the host are able to correlate their long molecular axes alignment parallel (or almost parallel) to the long axes of liquid crystal molecules, which leads to the high order parameter. Such behaviour of azo-dyes was also observed in other low molecular liquid crystals [18-20,23] and is in agreement with the theoretically predictions for binary mixtures [18, 27].

\section{Conclusions}

The influence of the dye molecular structure on the ability to the orientation in both side chain liquid crystalline polysiloxans and in low molecular weight liquid crystal was observed. It was also found that addition of the dye affects the behaviour of the mesogenic host in the vicinity of the phase transition. In some cases there exists a correlation between the change of the clearing temperature and the value of the dye order parameter.

Finally, it should be noticed that the results obtained for the dye-liquid crystalline polymer mixtures are not easy to univocal interpretation. Polysiloxanes used differ only with the length of the spacer. Nevertheless, in the mixtures with azo-dyes they behave very differently. It is especially seen at the nematic-isotropic phase transition, where the enormous increase in $T_{\mathrm{NI}}$ of LCP 1 after the dye addition is observed.

\section{Acknowledgment}

This work was supported by Research Project No. 5 P03B 02520 coordinated by the State Committee for Scientific Research.

\section{References}

[1] H. Finkelmann, G. Rehage, Adv. Polym. Sci. 60/61, 99 (1984)

[2] V.P. Shibaev, N.A. Plate, Adv. Polym. Sci. 60/61, 173 (1984).

[3] Liquid Crystal Polymers, Ed. N.A. Plate, Plenum Press, New York 1993.

[4] Side-Chain Liquid Crystal Polymers, Ed. C.B. Mc Ardle, Blackie, Glasgow 1989.

[5] V.D. Neff, in: Liquid Crystals and Plastic Crystals, Vol. 2, Eds. G.W. Gray, P.A. Winsor, Ellis Horwood, Chichester 1974, Ch. 9.

[6] G.H. Heilmeier, L.A. Zanoni, Appl. Phys. Lett. 13, 91 (1968).

[7] E. Wolarz, D. Bauman, J. Polym. Sci. B, Polym. Phys. 31, 383 (1993).

[8] E. Wolarz, D. Bauman, Liq. Cryst. 19, 221 (1995).

[9] H. Meier, in: Chemical Synthesis of Dyes, Vol. 4, Ed. Venkataraman, Academic Press, New York 1971, Ch. 7. 
[10] H. Finkelmann, G. Rehage, Macromol. Chem. Rapid Commun. 1, 31 (1980).

[11] Tables of Interatomic Distances and Configuration in Molecules and Ions, The Chemical Society, London 1958.

[12] A. Bondi, J. Phys. Chem. 68, 441 (1964).

[13] F.C. Saunders, L. Wright, M.G. Clark, in: Liquid Crystals and Ordered Fluids, Vol. 4, Eds. A.C. Griffin, J. F. Johnson, Plenum Press, New York 1984, p. 831.

[14] V. Zvetkoff, Acta Phys. USSR 10, 557 (1939).

[15] M.G. Pellat, I.H.C. Roe, J. Constant, Mol. Cryst. Liq. Cryst. 59, 299 (1989).

[16] J. Cognard, T. Hieu Phan, Mol. Cryst. Liq. Cryst. 68, 207 (1981).

[17] H.V. Ivashchenko, V G. Rumyantsev, Mol. Cryst. Liq. Cryst. A 150, 1 (1984).

[18] D. Bauman, Mol. Cryst. Liq. Cryst. 159, 197 (1988).

[19] D. Bauman, H. Moryson, J. Mol. Structure 404, 113 (1997).

[20] D. Bauman, J. Solczyński, E. Chrzumnicka, Acta Phys. Pol. A 92, 1151 (1997).

[21] A. Bloom, P.L.K. Hung, D. Meyerhofer, Mol. Cryst. Liq. Cryst. Lett. 41, 1 (1977).

[22] Z. Salamon, D. Bauman, Mol. Cryst. Liq. Cryst. Lett. 82, 115 (1982).

[23] D. Bauman, H. Moryson, J. Jadżyn, Acta Phys. Pol. A 85, 553 (1994).

[24] D. Bauman, E. Wolarz, Mol. Cryst. Liq. Cryst. Lett. 7, 161 (1990).

[25] P. Diot, J.K. Foitzik, W. Haase, Rev. Phys. Appl. 20, 121 (1985).

[26] D. Bauman, Z.-X. Fan, W. Haase, Acta Phys. Pol. A 80, 545 (1991).

[27] D.E. Martire, in: The Molecular Physics of Liquid Crystals, Eds. G.R. Luckhurst, G.W. Gray, Academic Press, London 1979, Ch. 10.

[28] P.J. Collings, Liquid Crystals, Princeton University Press, 1990, Ch. 9.

[29] R. Zentel, in: Liquid Crystals, Ed. H. Stegemeyer, Steinkopff, Darmstadt, Springer, New York 1994, Ch. 3. 